\title{
Dysphagia after stroke and its management
}

\author{
Rosemary Martino MSc PhD, Ruth E. Martin MHSc PhD, Sandra Black MD
}

$\mathrm{D}$ ysphagia is common after stroke, presenting in $55 \%$ of all hospital-admitted patients with acute disease, and, depending on the lesion site and volume, can linger as a chronic problem for years. ${ }^{1}$ It is estimated that every year in Canada there are 21000 new elderly patients with dysphagia after stroke and 200000 in the United States. ${ }^{2}$ Of these patients, as many as 10000 in Canada and 100000 in the US continue to experience dysphagia for months after the initial stroke event. The problem of dysphagia after stroke is expected to increase given the aging of our population.

Dysphagia after stroke is often underdiagnosed. ${ }^{3}$ Dysphagia needs to be detected early and managed effectively because the consequences of untreated dysphagia could be serious. ${ }^{2,4}$ Dysphagia has been associated with increased length of stay, malnutrition, dehydration and death. ${ }^{5}$ Reports of pneumonia in patients with dysphagia after stroke range from $7 \%$ to $33 \%$, with conservative estimates at $18 \% .^{1}$ Patients with dysphagia after stroke have a 3-fold increased risk for aspiration pneumonia, and this risk is markedly increased to 11 -fold in patients with confirmed aspiration on videofluoroscopy after stroke. ${ }^{1}$ Aspiration without a cough (silent aspiration) further increases the incidence of pneumonia to $54 \% .{ }^{6}$ This is problematic considering that silent aspiration occurs in up to two-thirds of patients with dysphagia after stroke. ${ }^{7}$

Evidence-based tools are now available to detect dysphagia early, even in patients with silent aspiration. New stroke guidelines in Canada are stressing early detection of dysphagia with validated screening tools. ${ }^{8}$ Similar guidelines exist in the US ${ }^{9}$ and the United Kingdom. ${ }^{10}$ These guidelines require that a trained clinician screen patients admitted with stroke or suspicion of stroke for dysphagia as soon as the patients are alert and able. A standardized tool must be used.

In an effort to standardize care for patients with stroke across all settings, the Toronto Bedside Swallowing Screening Test was recently developed. ${ }^{11}$ The test was conceptualized and developed on the premise that earlier detection of dysphagia by screening shortens the time to recovery and also improves overall patient health. ${ }^{2}$

There is now emerging evidence from one retrospective study to show that early detection of dysphagia reduces subsequent pulmonary complications in patients with stroke by threefold. ${ }^{4}$ Although, by design, retrospective data are limited because pneumonia surveillance could not be standardized, the reduction in pneumonia events following screening for dysphagia is compelling, especially because patients with pneumonia after stroke are at increased risk of dying (relative risk 5.4, 95\% confidence interval 3.29.0) and have a longer length of stay in hospital (mean 14 v. 5 d). ${ }^{4}$ Therefore, a screening intervention that reduces comorbidity and yet is brief and easy to administer, as well as psychometrically sound, is critical for provision of good patient care.

Currently, only $50 \%$ of patients with stroke in Canada are reportedly being screened for dysphagia. ${ }^{12}$ Considering that aspiration pneumonia is a preventable complication that continues to occur in $5.7 \%$ of patients, ${ }^{12}$ there is a need to increase the use of screening for dysphagia in the care of patients after stroke.

To maximize recovery and minimize negative consequences, early detection of dysphagia must be followed with proper management. ${ }^{2}$ Several approaches for management of dysphagia have become well established in clinical practice for stroke, including compensatory approaches such as enteral feeding, dietary modifications, posturing for feeding, advice on safe eating, and oral hygiene, as well as behavioural manoeuvres such as voluntary airway protection and effortful swallowing. ${ }^{13}$ The evidence suggests that use of these therapies more frequently would be beneficial. For example, when compared with usual swallowing therapy (5 sessions averaging 16 minutes each), high-intensity therapy (12 sessions averaging 24 minutes each) offered in the acute phase produced greater recovery of swal-

\section{KEY POINTS}

- Dysphagia after stroke is common, presenting in $55 \%$ of all hospitaladmitted patients with acute disease, yet is often underdiagnosed.

- Undetected dysphagia in patients after stroke increases rates of pneumonia by threefold.

- Early detection of dysphagia with screening followed by proper management reduces comorbidity, such as pneumonia. 
lowing ability and return to a normal diet at six months. ${ }^{14}$ Swallowing therapies can also focus on improving swallowing physiology through, for example, oral exercises, cold stimulation to the faucial pillars, transcutaneous neuromuscular electrical stimulation or olfactory stimulation..$^{13}$ Therefore, although data on efficacy remain limited, several recent randomized controlled trials have provided emerging evidence that general programs of therapy for dysphagia are associated with favourable outcomes. ${ }^{15}$

More recently, there is growing evidence that the neural network for swallowing is capable of experience-dependent plasticity. ${ }^{16}$ This in turn has fuelled the development of several experimental interventions for dysphagia. For example, sensory stimulation of the oropharynx with airpulse trains has been shown to increase resting swallowing rates in tube-fed patients with dysphagia after stroke ${ }^{17}$ and activate the cortical swallowing network in controls. ${ }^{18}$ Pharyngeal electrical stimulation has been reported to decrease aspiration, and increase cortical excitability, in patients with dysphagia after stroke. ${ }^{19}$ Although promising, these therapies are still under study. Randomized controlled trials are required to establish the therapeutic effects of these experimental swallowing interventions in terms of swallowing and stroke outcomes.

Dysphagia is common after stroke, and its presence can have serious consequences, such as pneumonia and even death. There is new evidence to show that early identification from screening and enthusiastic acute treatment will reduce these comorbidities and increase longterm functional recovery.

\section{References}

1. Martino R, Foley N, Bhogal S, et al. Dysphagia after stroke: incidence, diagnosis, and pulmonary complications. Stroke 2005;36:2756-63.

2. Martino R, Pron G, Diamant NE. Screening for oropharyngeal dysphagia in stroke: insufficient evidence for guidelines. Dysphagia 2000;15:19-30.

3. Martino R, Silver F, Newman A, et al. The usefulness of a national stroke registry to derive predictors for dysphagia [abstract]. Dysphagia 2007;22:402.

4. Hinchey JA, Shephard T, Furie K, et al. Formal dysphagia screening protocols prevent pneumonia. Stroke 2005;36:1972-6.

5. Altman KW, Yu GP, Schaefer SD. Consequence of dysphagia in the hospitalized patient: impact on prognosis and hospital resources. Arch Otolaryngol Head Neck Surg 2010;136:784-9.

6. Nakajoh K, Nakagawa T, Sekizawa K, et al. Relation between incidence of pneumonia and protective reflexes in post-stroke patients with oral or tube feeding. J Intern Med 2000;247:39-42.

7. Daniels SK, Brailey K, Priestly DH, et al. Aspiration in patients with acute stroke. Arch Phys Med Rehabil 1998;79:14-9.

8. Lindsay PBP, Bayley MM, Hellings CB, et al. Canadian best practice recommendations for stroke care (updated 2008). CMAJ 2008;179:E1-93.

9. Duncan PW, Zorowitz R, Bates B, et al. Management of adult stroke rehabilitation care: a clinical practice guideline. Stroke 2005;36:e100-43

10. Intercollegiate Stroke Working Party. National clinical guidelines for stroke. 2nd ed. London (UK): Royal College of Physicians; 2004.

11. Martino R, Silver F, Teasell R, et al. The Toronto Bedside Swallowing Screening Test (TOR-BSST): development and validation of a dysphagia screening tool for patients with stroke. Stroke 2009;40:555-61.

12. Canadian Stroke Network. The quality of stroke care in Canada. Ottawa (ON): The Network; 2011.

13. Logemann JA. Dysphagia: evaluation and treatment. Folia Phoniatr Logop 1995;47:140-64.

14. Carnaby G, Hankey GJ, Pizzi J. Behavioural intervention for dysphagia in acute stroke: a randomised controlled trial. Lancet Neurol 2006;5:31-7.

15. Foley N, Teasell R, Salter K, et al. Dysphagia treatment post stroke: a systematic review of randomised controlled trials. Age Ageing 2008;37:258-64

16. Martin RE. Neuroplasticity and swallowing. Dysphagia 2009; 24:218-29.

17. Martin RE, Theurer J, Czachorowski K, et al. Effects of oropharyngeal air-pulse parameters on dry swallowing rates in patients with dysphagia following stroke [abstract]. Dysphagia 2010;25:366-7.

18. Sörös P, Lalone E, Smith R, et al. Functional MRI of oropharyngeal air-pulse stimulation. Neuroscience 2008;153:1300-8.

19. Fraser C, Power M, Hamdy S, et al. Driving plasticity in human adult motor cortex is associated with improved motor function after brain injury. Neuron 2002;34:831-40.

Competing interests: Rosemary Martino has received funds from Nestlé Health Science for travel expenses related to providing consultation. Ruth Martin has received royalties for the Swallowing Air-Pulse Therapy system, and her institution has received a Proof of Principle Phase II grant from the Canadian Institutes of Health Research and has patents on oral devices related to Swallowing Air-Pulse Therapy. Sandra Black has had financial relationships in the form of contract research funds to her institution from Pfizer (Wyeth Research), Pfizer Canada, GlaxoSmithKline, Lundbeck, Roche Pharmaceuticals and Novartis Pharmaceuticals; speaker's honoraria for CME from Pfizer and Eisai; and honoraria for ad hoc consulting from Pfizer, Elan and Eyeth Pharmaceuticals, Bristol-Myers Squibb, Novartis and Roche Pharmaceuticals

Affiliations: Rosemary Martino is with the Department of Speech-Language Pathology, University of Toronto, and the Toronto Western Research Institute, University Health Network Toronto, Ont.; Ruth E. Martin is with the School of Communication Sciences and Disorders, and the Departments of Physiology and Pharmacology, and Otolaryngology, University of Western Ontario, London, Ont.; Sandra Black is with the Department of Medicine, Division of Neurology, Sunnybrook Health Sciences Centre, University of Toronto, and the Brain Sciences Program, Sunnybrook Research Institute, Toronto, Ont.

Contributors: All of the authors contributed to drafting and revising the manuscript and approved the final version submitted for publication.

Funding: Rosemary Martino was supported by a Canadian Institutes of Health Research New Investigator Fellowship Award in Aging. 